\title{
Analysis of Tungsten Melt Layer Motion and Splashing under Tokamak Conditions at TEXTOR
}

J.W. Coenen ${ }^{1 *}$, V. Philipps ${ }^{1}$, S. Brezinsek ${ }^{1}$, B. Bazylev ${ }^{2}$, A. Kreter ${ }^{1}$, T. Hirai ${ }^{3}$, M. Laengner ${ }^{1}$, T. Tanabe ${ }^{4}$, Y. Ueda ${ }^{5}, \mathrm{U} \mathrm{Samm}^{1}$ and the TEXTOR-Team

${ }^{1}$ IEF-4 (Plasmaphysik), Forschungszentrum Jülich GmbH, Association EURATOM-FZJ, Partner in the Trilateral Euregio Cluster, 52425 Jülich, Germany, ${ }^{2}$ Institut für Hochleistungsimpuls und Mikrowellentechnik, Forschungszentrum Karlsruhe GmbH, Association Euratom-FZK, 76021 Karlsruhe, Germany, ${ }^{3}$ ITER Organization CS 90 046-13067 St Paul Lez Durance Cedex, France, ${ }^{4}$ Interdisciplinary Graduate School of Engineering Science, Kyushu University, Hakozaki 6-10, Higashiku, Fukuoka 812-8581, Japan, ${ }^{5}$ Graduate School of Engineering, Osaka University, Osaka 565-0871, Japan

* j.w.coenen@fz-juelich.de

\begin{abstract}
Behavior and characteristics of tungsten under impinging high heat-fluxes are investigated in view of the material choices for future devices such as ITER and DEMO. Experiments have been performed in the edge of the TEXTOR tokamak to study melt-layer motion, macroscopic melt layer erosion as well as the changes of the material properties. The parallel heat-flux ranges around $q_{\|} \sim 45 \mathrm{MW} / \mathrm{m}^{2}$ allowing samples at an impact angle of $35^{\circ}$ to be exposed to $20-$ $30 \mathrm{MW} / \mathrm{m}^{2}$. Melt-layer motion perpendicular to the magnetic field is observed following a Lorentzforce originating from thermoelectric emission of the hot sample. Up to $3 \mathrm{~g}$ of tungsten are redistributed forming mountain like structures at the edge of the sample. The typical melt layer thickness is $1-1.5 \mathrm{~mm}$. Those hills are particularly susceptible to even higher heat-fluxes of up to the full $q_{\|}$. Locally the temperature can reach up to $6000 \mathrm{~K}$, high levels of evaporation are causing significant erosion in form of continuous fine-spray $\left(\sim 1 \cdot 10^{24}\right.$ atoms $\left.m^{-2} s^{-1}\right)$. Vaporshielding is occurring and hindering the further heating of the samples. In addition the formation of ligaments and splashes occurs several times during the melt phase ejecting droplets in the order of several $10 \mu \mathrm{m}$ up to $100 \mu \mathrm{m}$ probably caused by a Kelvin-Helmholtz instability evolving in the melt. In terms of material degradation several aspects are considered: formation of leading edges by redistributed melt, bubble formation and re-crystallization. Bubbles are occurring in sizes between $\mu \mathrm{m}$ and $200 \mu \mathrm{m}$ while recrystallization increases the grain size up to $1.5 \mathrm{~mm}$. The power handling capabilities are thus severely degraded. Melting of Tungsten in future devices is highly unfavorable and needs to be avoided especially in light of uncontrolled transients and possible unshaped PFCs
\end{abstract}

\section{Introduction}

Plasma Wall Interaction (PWI) is one of the main aspects to be considered toward ITER and a future fusion reactor. With the necessity of long life time and favorable thermomechanical properties High - Z materials are now the materials of choice. Tungsten due to is low sputtering yields, highest melting point $(3695 \mathrm{~K})$ and its rather benign behavior after neutron irradiation was chosen as part of the ITER divertor design and is the material used for the activated phase. It will replace carbon which, suffering from the issue of strong erosion and tritium co-deposition, is probably not acceptable due to tritium inventory limits in C layers. Tungsten however, apart from its favorable properties, is leading to additional constraints in particular for plasma operation. The possibility of melting under uncontrolled conditions and the associated material redistribution and possible material loss place limits on the plasma performance and the material lifetime. When talking about power-handling excessive heat loads on plasma facing components (PFCs) due to loss of plasma control or leading edges are a critical issue when operating a fusion power-plant with a full metal wall. Development of melt layers, motion and ejection of melt can drastically decrease the lifetime of the PFCs while leading to strong plasma contamination and degradation of the power-handling capabilities. In contrast to the low- $\mathrm{Z}$ materials like Carbon only minuscule amounts of tungsten (concentration, $10^{-5}$ ) can be tolerated in the plasma core to avoid cooling and stop of the fusion process . For ITER steady 
state heat loads for a tungsten divertor plate are expected to range around $5-10 \mathrm{MW} / \mathrm{m}^{2}$ under normal operation conditions in the non-activated phase, loss of positioning control or additional transients as well as possible misalignment of target mono-blocks can thus lead to melting $[1,2]$. Studies on melt-layer motion have been performed in electron and ion beam facilities $[3,4,5,6]$ showing significant melt motion, splashing and changes in the material structure and their power handling capabilities. Few results exist regarding behavior under tokamak conditions, including magnetic fields, large currents through the PFC surfaces and various power impact scenarios (Steady state, ELMs, VDEs or disruptions) as well as high temperature erosion. Recent experiments have been performed in TEXTOR focussing on the melt-layer motion and the material properties after exposure. Regarding high heat-loads aspect of vapor-shielding have to be considered as seen in [3] which have been observed for transient events in QSPA ELM simulation. For pure tungsten almost $50 \%$ of the impinging heat-flux can be observed. Under tokamak conditions this aspect has to be discussed with respect to melt incidents under realistic conditions (fig. 2 \& section 3.2). In this paper the observations and conclusions drawn from melt experiments with tungsten samples at TEXTOR will be presented comparing also to previously obtained results $([7,8])$. The paper starts by describing the experimental setup followed by the presentation of the results which are subsquently summarized.

\section{Experimental Setup and Diagnostics}

Experiments have been performed by introducing a limiter into the deuterium plasma by means of the PWI-test facilities [9] at TEXTOR $(\mathrm{R}=1.75 \mathrm{~m} \mathrm{a}=0.47 \mathrm{~cm})[10]$. The plasma parameters are: $I_{p}=350 k A, B_{T}=2.25 T, \bar{n}_{e}=3.5 \times 10^{19} \mathrm{~m}^{-3}, P_{N B I}=1.2 \mathrm{MW}$. The limiters were moved stepwise $\left(\Delta_{r} \sim 0.2 \mathrm{~mm}\right)$ towards the LCFS and beyond until melting occurred at $\sim 46.2 \mathrm{~cm} .\left(T_{e}(a) \sim 70 \mathrm{eV}, n_{e}(a) \sim 1 \times 10^{19} \mathrm{~m}^{-3}, q_{\|} \sim 70 \mathrm{MW} / \mathrm{m}^{2}\right)$. The limiter is equipped with a thermally isolated tungsten on either side (fig. 1). Multiple exposures were performed. Single exposures are described in $[7,11]$. The average temperature rises up to $3500 K$ with an average heat flux of $10 \mathrm{MW} / \mathrm{m}^{2}$ while the upper part of the can receive up to $45 \mathrm{MW} / \mathrm{m}^{2}$ for leading

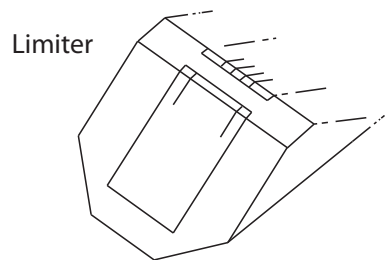

Figure 1: (Left) Limiter setup (Right) Diagnostic views (Circle $=$ pyrometer). Gray areas indicate tiles $(3.5 \mathrm{~cm} \times 5.5 \mathrm{~cm} \times 0.2 \mathrm{~cm})$.

edges. The typical heat flux is $\sim 20 \mathrm{MW} / \mathrm{m}^{2}$ for a duration of $5 \mathrm{~s}$, peak temperatures from IR measurements reach $(4000-6000) K$. Vapor-shielding leads to a constant temperature level and typically a decrease of heat-flux reaching the target in the flattop melting phase (fig. 2). In the case of multiple exposures evolution of hot-spots has been observed. Temperatures above $5000 K$ can be reached locally. An extensive set of diagnostics is used $[12,13]$. Temperature measurements are performed via a single color pyrometer (1 spot $3.25 \mu m$ ) (fig.1) and a near infrared camera $((1.0-1.7) \mu m)$. Tungsten emissivity dependencies are taken into account $[14,15]$. Spectroscopic data is available from core VUV data (W continuum at $\sim 5 n m$ ) as well as from the local emission of the $400.8 \mathrm{~nm}$ WI line (Overview Spectrometer). 2D video cameras (20ms time resolu-

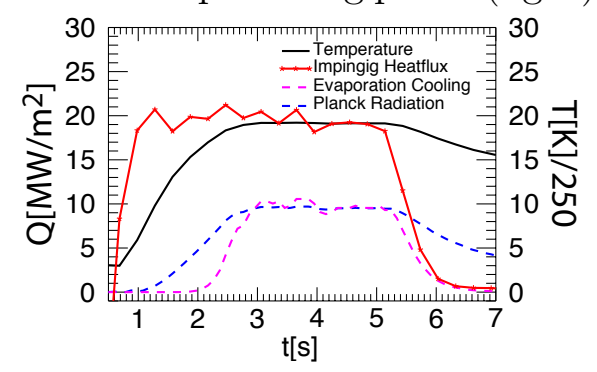

Figure 2: Impinging heat flux and Temperature (\#112054) tion), from the top as well as from the side (fig.1), are used to observe the WI influx and the particle ejection. Melt-layer motion has been observed with the $2 \mathrm{D}$ camera system. The measurement of the thermoelectric emission is performed by means of a $0.22 \Omega$ resistor. In order to perform visual inspection in the far SOL $(\mathrm{r}=48 \mathrm{~cm})$, the $\mathrm{H} \alpha$ emission of the plasma is used as a light source. Ex-situ analysis is using large scale profilometry $(1 \mu \mathrm{m}$ height resolution, $0.3 \mathrm{~mm}$ lateral accuracy) and scanning electron microscopy (SEM). 


\section{Results and Measurements}

The measurements presented here are indicative for high heat loads events under either steady state conditions including loss of detachment or positioning control in ITER [2] causing strong increase in heat-flux to the divertor targets. Transient events like ELMS, and VDEs are discussed elsewhere [3, 4].

\subsection{Melt Layer Evolution}

For the experiments performed here we typically consider the following forces: Plasma pressure, $j \times B$ force and recoil pressure. The $j \times B$ force originates from thermoelectric emission following the RichardsonDushman equation [16, 7]. Meltlayer motion as observed in the Quasi Stationary Plasma Accelera-

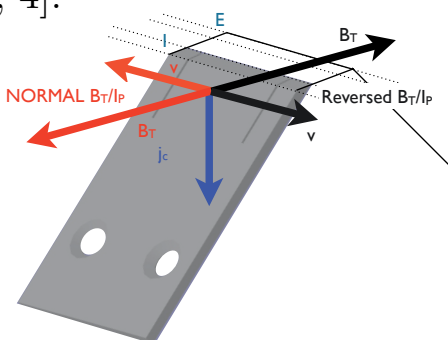

Figure 3: (Left) Directions of motion due to the thermoelectric-emission and B-Field (Right) Measured current (Richardson-current in red) tor (QSPA) facilities $[3,4,5,6]$ is driven by the high plasma pressure $\left(\sim 10^{5} \mathrm{~Pa}\right)$. Few studies have in addition considered Lorentz-forces $\left(F_{j \times B}\right)$ due to thermoelectric emission and those have been done at medium B-fields (1.4T) [17]. The driving force which has been identified as cause for bridging the castellation is the inertia of the melt at the gap edge competing with the surface tension, melt layer splashing is assumed to be caused due to high velocity shears leading to Kelvin-Helmholtz instabilities [5, 4]. During tokamak experiments the situation is quite different. In ITER the plasma pressure may reach $10^{3} \mathrm{~Pa}$ and even higher values during ELMs $\left(10^{4} \mathrm{~Pa}\right)[18]$, hence only during ELMs a significant plasma pressure could be accessible to drive the melt. Under TEXTOR conditions $F_{p}$ is typically $200 P a$ and thus small in comparison to $F_{j \times B}$ which moves the material along the sample edge, perpendicularly to the magnetic field. The typical situation as given for the performed experiments is thus given in figure 3 for both directions of the toroidal field. The ratio is determined by the melt layer thickness. The thermoelectric current is measured at TEXTOR and amounts to up to $40 \mathrm{~A}$ while at $2600 \mathrm{~K}$ starts do deviate from the richardson law figure 3. This accounts for a molten surface of about $3-4 \mathrm{~cm}^{2}$ consistent with previous results [11]. This current can lead to up to $3 \mathrm{kN} / \mathrm{m}^{2}$ for a $1 \mathrm{~mm}$ melt layer, which is large in comparison to $F_{p}$ while still small when compared to the QSPA studies. The force is large enough to move the melt against gravity as was observed in previous experiments [7]. Saturation of the electron current occurs due to space charge which limits the electron current [19] flowing through the target. The recoil pressure due to the evaporation is when considering the whole sample on average small compared to the Lorentz-force and will be discussed only as mechanism to drive the local melt layer instability (section 3.3) due to local boiling or evaporation (section 3.2). In terms of future devices the balance between steady state heatloads and transients events is becoming important. Melt-layer motion may be dominantly driven either by plasma pressure during ELMs or Lorentz-forces during long term melting. Control of the $j \times B$ forces was shown [20] for a liquid limiter but seems doubtful for an all metal castellated wall. Extrapolation to ITER can only be done via further modeling.
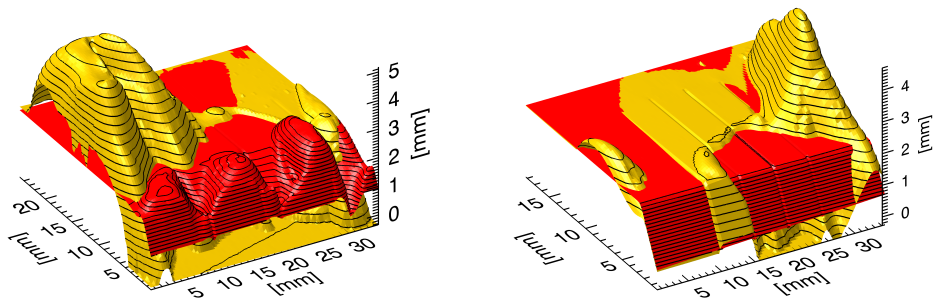

Figure 4: (Left - 2L), (Right - 2H) Profiles before (red) and after exposure (golden) idently moving large portions of the material. The samples are embedded into a massive graphite limiter head, thus the material motion stops at the much colder borIn order to display the effects of the melt-layer motion and the operating $j \times B$ force figure 4 displays the profilometric measurements before and after multiple exposures. Depending on the side of the limiter on which the sample was mounted (fig. 1) the force either operated to the left or right hand side of the sample (fig. 3) ev- 
der. Strong hill structures evolved with an increase in height of up to $3 \mathrm{~mm}$. A similar situation will occur during local melt events even with full metal components such as the ITER Baffle or a full W divertor like in JET or future devices.
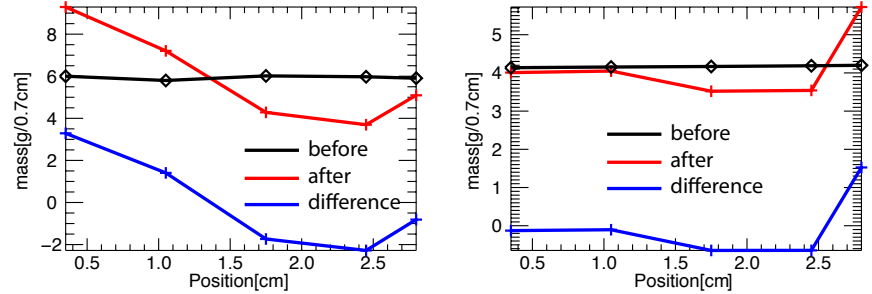

Figure 5: (Left - 2L), (Right - 2H) Mass motion per target segment As displayed in figure 4 the material is mostly originating from the front edge of the sample, in this case the hottest part and is then moved following the $j \times B$ force to the outer outer area of the sample. In case of Sample $2 \mathrm{H}$ multiple exposures under forward field conditions have moved material to one side of the sample while an additional last exposure with reversed field has caused the little additional hill visible (fig.4). The steady state melt motion is clearly dominated by the Lorentz-force causing topology changes severely impacting the material distribution on the sample. In terms of mass motion it can be clearly deduced when considering 5 slices along the sample as depicted in figure 5. For sample $2 \mathrm{~L}$ up to 2 grams are redistributed into the large hill, while for the sample $2 \mathrm{H}$ it seems in terms of mass motion far less redistribution is taking place along the sample edge, still locally hill structures are formed, causing much more easy access for impinging heat-flux. The nominal impact angle is changing in parts of the sample up to $90^{\circ}$. This can cause a strong increase in temperature and local melting due to the impact of the full parallel heat-flux $(\sin (90) / \sin (35) \sim 2)$. Leading egdes have a significant impact on the heat-flux onto the target. It was measured that the heat flux on the total sample can be on average quite low $\left(\sim 10 \mathrm{MW} / \mathrm{m}^{2}\right)$, the leading edge formed during previous melt events in a receded area of the sample is however receiving up to $\sim 40 \mathrm{MW} / \mathrm{m}^{2}$ until the edge is molten and the material redistributed. This may warrant the observation that plasma-shaping is taking place, but the opposite effect is the case. While in some areas material is moved away from the hottest areas in other previously unexposed areas hill-structures can be maintained and are subject to further increase heat-flux. The material redistribution is severely degrading the power-handling capabilities by strongly changing the material distribution, thickness and orientation as well as angles with respect to the field lines. With respect to ITER, heat-flux on unshaped tiles is considered as a possible origin of melt layer development. With a misalignment of about $0.3 \mathrm{~mm}$ and $10 \mathrm{MW}$ of perpendicular heat-flux melting will occur [1]. For ITER one would have $(\sin (90) / \sin (3) \sim 20)$. It seems likely that either a loss of positioning control or otherwise increased heat-flux causes steady state like melting and subsequently damage the tiles. Favorable plasma shaping seems unlikely given recent experience, thus the damage will further facilitate melt layer development. However, modeling of all forces including especially transient loads is necessary. The motion of melt will be given by a combination of $j \times B$ forces and pressure driven ELM like loads and thus might cause a movement along the poloidal direction and generally outwards from the local impact position. For the TEXTOR experiment only small mass losses have been observed. In most cases such as sample $2 \mathrm{H}$ the loss is in the $m g$ range while for events with strong evaporation and loss of loosely bound layers up to $0.2 g$ are lost. A larger exposed sample area (ITER baffles or divertor plates) may well show a mass loss of several grams and thus cause the termination of even an ITER plasma as demonstrated in recent ASDEX-Upgrade experiments [21]

\subsection{Tungsten Erosion}

Tungsten Erosion by means of several different mechanisms may occur during high temperature expsosures. While so far considering $\mathrm{W}$ erosion by means of physical sputtering even under high temperature conditions [8] evaporation and even macroscopic loss of melt by fine-spraying or splashing as typical for the QSPA facilities $[22,11,3]$ is observed. In the course of the presented experiments apart of the underlying sputtering contribution, particle ejection is observed during all melt exposures. Figure 6 is showing both phenomena during a previous experiment. Clearly two phenomena are visible. 
On the left hand side so-called fine-spraying occurring continuously during the melting is shown while on the right hand side an event considered to be splashing is displayed. Strong local heating and evaporation seem to drive the fine-spray and subsequently the melt layer instability leading to splash events. The spray is ejected at velocities of $\sim 1 \mathrm{~m} / \mathrm{s}$ while the splashes are significantly faster $(\sim 3 \mathrm{~m} / \mathrm{s})$. Two kinds of droplets may be distinguished, the small ones called spray $(\sim 4 \mu \mathrm{m}$ droplets) and the ones being present in the splashing with droplets between 20 and $100 \mu \mathrm{m}$ in size, which can in a single event lead to a disruption. Both phenomena represent a significant $\mathrm{W}$ source

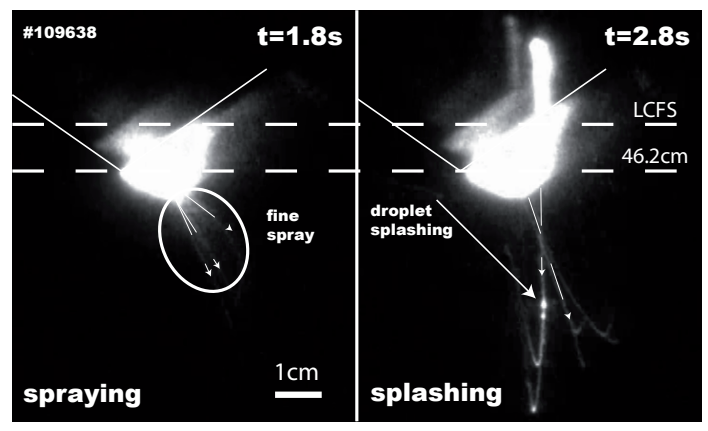

Figure 6: Melt layer ejection, spraying(left) and splashing(right), LCFS is indicated [11]. as discussed below before describing the droplets and possible production mechanisms (section 3.3). From local spectroscopy a measure for the local W source (fig. 7) can be derived and compared to the core emission (fig. 7).Figure 7 shows the comparison between two melt discharges, \#112060 ending in a disruption, \#112062 without. In the first case a strong increase of the $\mathrm{W}$ source around 2 seconds is observed while for the non disrupting case a slow rise to about the same level is seen. The source is dominated by the so called fine-spraying, while in both cases several small spikes are visible, connected to splash events during the melting. Due to the lifetime of the droplets (section 3.3) they leave the local observation volume before, if ever, completely disintegrating. When considering figure 7 this becomes clearly visible. The local source rises and is quite steady over most of the discharge while the core VUV emission shows some spikes for each of the larger droplets emitted from the melt. Regarding the increase of core emission with the fine-spraying one can observe that the core emission clearly scales with the local W source in the beginning of the discharge. When comparing both discharges with and without disruption one effect becomes visible, depending on the amount of $\mathrm{W}$ emitted by the droplets the subsequent cooling can lead to a decrease of core $\mathrm{W}$ emission and thus a cooling or accumulation in the core, for the non disrupting case the emission after the last droplet is significantly lower compared to the amount before. In terms of quantitative results one can now compare to high temperature erosion as describe in [8].
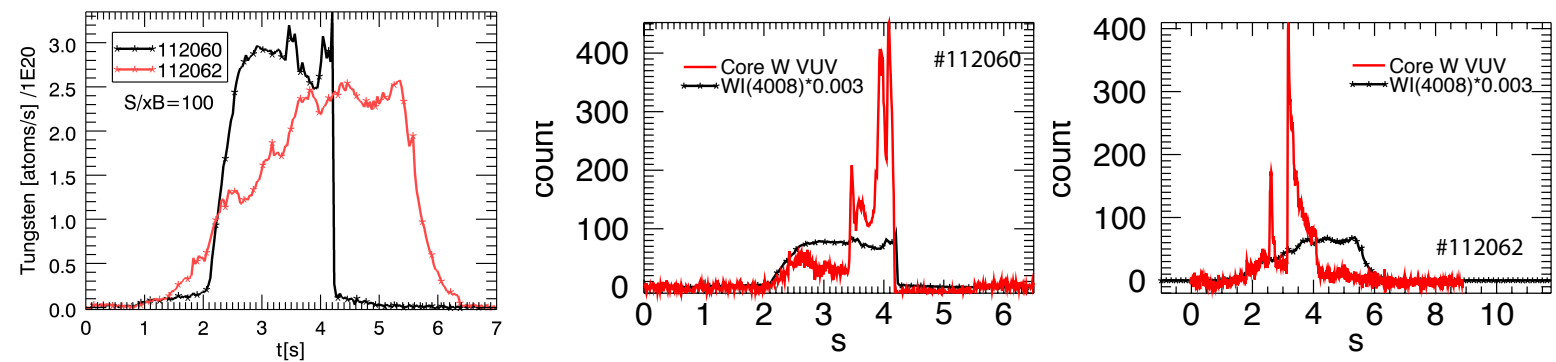

Figure 7: (Left) Local W source from WI (400.8nm) emission. (Right ) Time traces of local and core emission compared

In both cases the amount of $\mathrm{W}$ releases reaches between 2.5 and $3 \cdot 10^{20}$ atoms/s which is, assuming a small area of about $(1-4) \mathrm{cm}^{2}$ for the melt and interaction surface and an $\mathrm{S} / \mathrm{XB}$ value of 100 [23], still orders of magnitude larger $\left(\sim 10^{24} \mathrm{~m}^{-2} \mathrm{~s}^{-1}\right)$ than for typical high temperature erosion as deduced previously $\left(4 \cdot 10^{21} \mathrm{~m}^{-2} \mathrm{~s}^{-1}\right)$ [8]. This is compatible with the assumption of strong local heating and thus strong local evaporation. Figure 8 shows the W evaporation flux as well as the vapor pressure for sample temperatures between $2500 \mathrm{~K}$ and $5000 \mathrm{~K}$. With a hot spot temperature lying in the range of $4000 \mathrm{~K}-4500 \mathrm{~K}$ the evaporation flux lies at $\sim 10^{24}$ atoms $/ \mathrm{m}^{-2} \mathrm{~s}^{-1}$ which is well in the range seen from local spectroscopy. The vapor pressure for this case can reach $2000 P a$ and is similar to the typical melt layer driving forces. This of course covers only the continuous fine-spraying events, while droplets can transport on a short timescale far more material from the surface. Vapor shielding 
compatible with the observed $\mathrm{W}$ influx due to the fine-spraying is observed and can account for $50 \%$ of the impinging heat-flux (fig. 2) leading to the flat temperature curve (fig. 8). Using the MEMOS [24, 25] code one can accurately reproduce the Temperature and heat flux evolution observed in the measurements, giving a clear indication towards the validity of the modeling and the accuracy of the data given for the observed phenomena.Modeling of the melt-layer motion as observed in 3.1 can thus be performed for an extrapolation towards future devices in case of transient and steady state melt exposures.
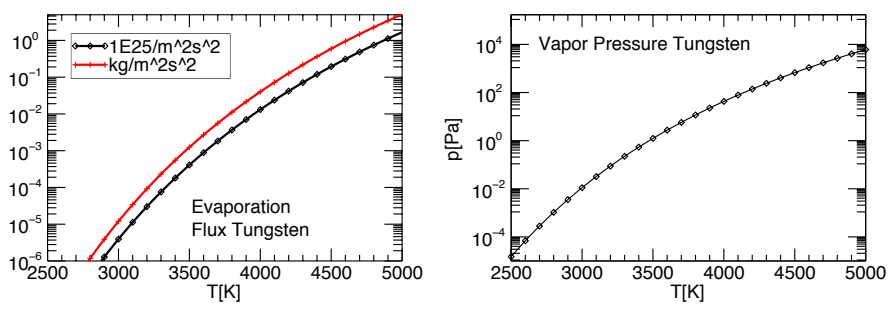

Figure 8: Evaporation flux and vapor pressure Already from the spectroscopic data the transport of $\mathrm{W}$ to the core could be seen, several larger droplets can lead to a strong increase in core $\mathrm{W}$ emission. In terms of radiative power loss this can even cause a disruption (\#112060 ,fig. 7). For a typical non melting discharge the total radiated power-fraction lies at about $30 \%$ with a total power of $1.5 \mathrm{MW}$. One observes that fine-spraying adds another $50-100 \mathrm{KW}$ of constant core-radiation to, droplets however can cause significantly more disruptive events.
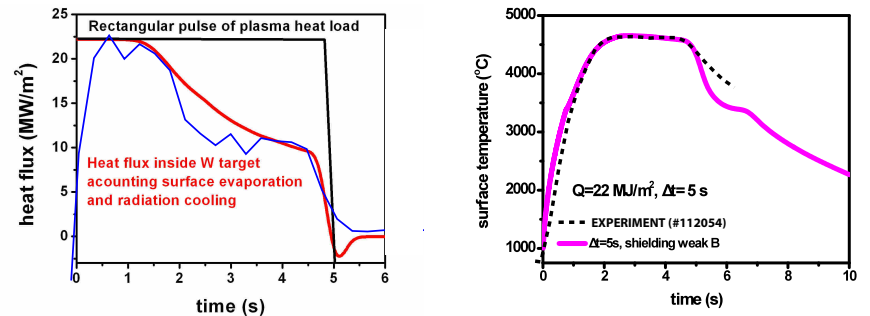

Figure 9: Heat flux and temperature Data vs. MEMOS [24, 25] A single droplet of $30 \mu \mathrm{m}$ size if completely radiating in the core will give rise to additional $200 \mathrm{~kW}[26,27,28]$ and larger droplets can cause the radiative collapse $(\# 112060 \mathrm{t}=4 s)$ or come close to it (\#112062 $\mathrm{t}=3.2 \mathrm{~s})$. This means that in order to operate a high power high performance discharge probably spraying but for sure splashing of droplets has to be avoided. As stated in [21] for a typical ITER plasma an amount of $1 \mathrm{~g}$ of $\mathrm{W}$ may be sufficient to cause fatal accumulation in the core. This means that based on this results even a small of steady state melting can cause a disruption.

\subsection{Fine-Spray, Droplets and Ligaments}
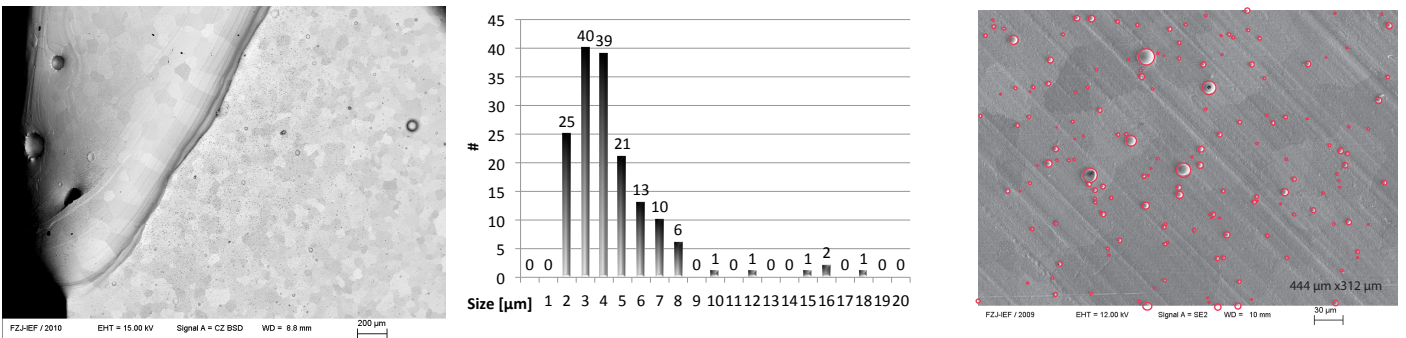

Figure 10: Droplet and Bubble / Impact crater after multiple melt exposures

Droplets and either bubble bursts or impact craters can be found close to the molten surface area, and on the melt as can be seen in figure 10. Those phenomena can be found during all of the recent melt exposures and are directly related to the $\mathrm{W}$ source of spraying and splashing as seen above. Material properties such as purity may play a role here and will be studied in future exposures. Previous melt experiments with less strong local heating have not shown this droplet evolution [7,8]. The typical redeposited droplet has a diameter of $4 \mu m$ (fig.10, center) while smaller droplets can hardly be found on the surface. Droplets in the range of 10 to $20 \mu \mathrm{m}$ do exist on the studied sample while the largest droplets are found as single occurrences. Bubbles or impact crates exist up to a size of $200 \mu \mathrm{m}$. Even though the droplets are rather small and the parallel heat-flux can be as high as $60 \mathrm{MW} / \mathrm{m}^{2}$ the lifetime of a $6.25 \mu \mathrm{m}$ droplet can reach up to $0.02 \mathrm{~s}$ and a $100 \mu \mathrm{m}$ droplet will live almost half a second [21]. This means that large droplets as observed can leave the local observation volume (fig. 7), and partly reach the plasma center or farther part of the vessel, while smaller 
droplets are probably redeposited locally (fig. 10). A more detailed transport analysis regarding the droplet impact on the core emission is required for further extrapolations [21].

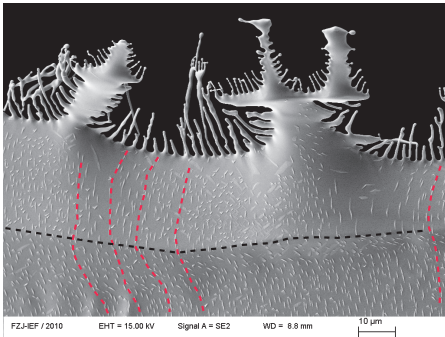

Figure 11: Ligament structures after exposure Part of the splashing and spraying events is the evolution of ligament like structures which seem to have a quite regular pattern probably following the plasma flow and or the melt motion on the surface and beyond. As predicted in [29, 30] instabilities are evolving due to the plasma flow and the melt-layer motion and the velocity shear between them. Critical levels of velocity shear still maybe reached or recoil may trigger splashing as described in [22]. The droplets are typically expected to be comparable in size with the experimentally observed splashing events while smaller droplets are not covered. The ligaments are forming on the surfaces and are following the plasma flow and velocity shear as can also be seen by the pattern in figure 11. Droplet evolution and splashing with ligaments thus seems to be rather well explained by the Kelvin Helmholtz instability of the surface. Smaller droplets (fine-spray) are more likely evolving due to local evaporation of W connected with evolution of bubbles close to the surface and their bursting [31] which is consistent with the local source.

\subsection{Material-Evolution}
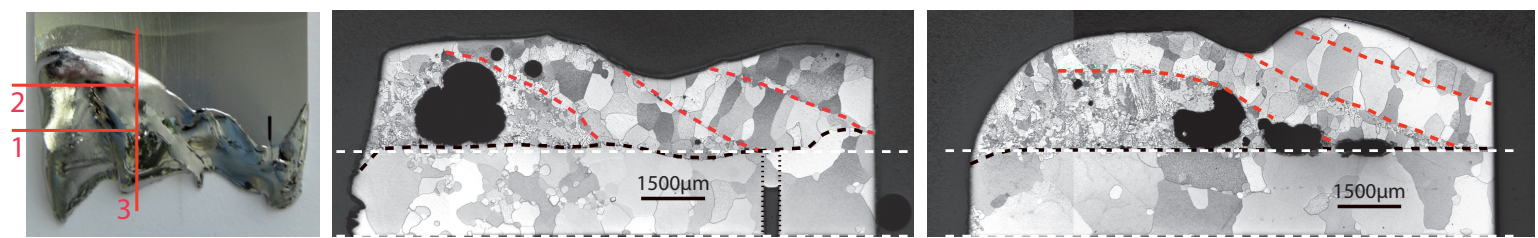

Figure 12: (Left) Cutting Plan 'Sample 2L' (cf. Figure 4 ) (Right) (1) and (2), layer evolution (red), original sample thickness (white), gap position and evolved surface (black)

In the course of material redistribution several effects play a role changing the material properties. Re-crystallization, boiling and layer formation are crucial to understand the material properties of the exposed samples. The evolution of the hill like structure is clearly seen in figure 12 in conjunction with bubble like structures close to the surfaces (small) and deep in the material. The different melt events can be distinguished by the different sized grain sizes, which are occurring due to the different thermal history of the layer. The lower ones experiencing less long exposure and lower initial heat-fluxes, while the later ones originate from leading edges and are re-solidifying already under higher temperature exposure. Bubbles are observed along all boundaries including the upper surface boundary, consistently with the local effect of evaporation and bubble bursting described above. The large irregular melt bubbles seem to have evolved in the course of the whole exposure cycle. while not being able to reach the surface for relaxation. In figure 12 the bubbles on the surfaces can be seen in the upper melt layer. A very critical point is the re-crystallization of the material already observed in previous experiments and seen in figures 12 . Prolonged exposure to high temperatures and heat-loads causes large grain growth up the range of $\mathrm{mm}$. This together with bubble growth makes the material severely brittle and changes significantly the thermal properties leading to a potentially large degradation in powerhandling capabilities. Highly refined and doped $\mathrm{W}$ materials have to be studied further considering the material and grain structure changes under severe heat-fluxes.

\section{Conclusions}

From the presented results, conclusions can be drawn with particular focus on material choices for high heat-load components in future devices and their effect on machine performance. High-Z materials in the worst case could prohibit plasma operation assuming strong plasma contamination. Evidently melting of plasma facing components is one of the most severely restraining aspects for future devices. Melt motion driven under steady state conditions by jxB forces can cause severe changes in topology and lead to an increase 
of heat-loads by a factor of up to 20, when considering leading edges with a assumed steep impact angle, causing further melting. Beneficial plasma-shaping seems unlikely. Even though material is moved away from the heat affected parts, new hills or leading edges are formed with height of possible several $\mathrm{mm}$ and angles of up to $90^{\circ}$ with respect to the field lines. Those leading edges represent a significant source for $\mathrm{W}$ evaporation (spraying) (up to $10^{24} \mathrm{~m}^{-2} \mathrm{~s}-1$ ) and splashing thus contaminating the plasma. A $30 \mu \mathrm{m}$ droplet can cause up to $200 \mathrm{~kW}$ additional core radiation. Initial modeling of the $\mathrm{W}$ splashing and spraying indicate good agreement with the experiments and have to be extrapolated towards ITER. The mechanism causing the spraying seems to be connected to local bubble boiling and thus strong evaporation. Splashing is more or less driven by a Kelvin-Helmholtz instability facilitated by the melt motion, plasma flow and evaporation recoil pressure. Further modeling needs to be performed. Due to the strong evaporation vapor shielding is observed, keeping almost $50 \%$ of the impinging $20 \mathrm{MW} / \mathrm{m}^{2}$ from reaching the surface. The result is twofold, as described above the plasma is strongly contaminated, on the other hand further increase of the surface temperature is stopped. For ITER vapor shielding during an ELM may be tolerable, but continuous spray at a rate of up to $10^{24} m^{-2} s-1$ would amount to several grams per second considering a larger affected area $\left(\sim 1 \mathrm{~m}^{2}\right)$. Regarding the material properties it seems possible to manufacture suitable materials with better stress resistance or thermal conductivity [6], it seems however unlikely to preserve those characteristics once exposed to strong local heat-loads and melting. Melting and melt layer spraying and splashing have to be avoided. Otherwise a strategy has to be devised for dealing with the severely degraded component lifetime and power-handling capability, should the observed effects be present in future devices.

\section{Acknowledgements}

This work, supported by the European Communities under the contract of Association between EURATOM/FZJ, was carried out within the framework of the EFDA Task Force on Plasma Wall Interactions. The views and opinions expressed herein do not necessarily reflect those of the European Commission.

\section{References}

1] Pitts, R. A. et al. Journal of Nuclear Materials - PSI 2010. Incl. priv. comm.

2 Neu, R. L. IEEE Transactions on Plasma Science, 38 (2010), 3 PART 1, 453-460.

3. Garkusha, I. et al. Journal of Nuclear Materials, 390-391 (2009), 814-817.

4 Klimov, N. et al. Journal of Nuclear Materials, 390-391 (2009), 721-726.

5] Bazylev, B. et al. Fusion Engineering and Design, 84 (2009), 2-6, 441-445.

6] Linke, J. Fusion Science And Technology, 53 (2008), 2T, 278-287.

7. Sergienko, G. et al. Physica Scripta, T128 (2007), 81-86.

8. Sergienko, G. et al. Journal of Nuclear Materials, 363-365 (2007), 96 - 100.

9. Schweer, B. et al. Fusion Science and Technology, 47 (2005), 2, 138-145.

[10] Neubauer, O. et al. Fusion Science And Technology, 47 (2005), 2, 76-86.

11. Coenen, J. W. et al. Journal of Nuclear Materials - PSI2010, (2011).

12] Brezinsek, S. et al. Fusion Science And Technology, 47 (2005), 2, 209-219.

13] Pospieszczyk, A. Physica Scripta, T119 (2005), 71-82.

[14] Sergienko, G. Priv. Communications, (2010).

15 Lide, D. R. and Frederikse, H. P. R., editors. crc handbook. CRC Press (1994-95).

16. Richardson, O. The emission of electricity from hot bodies. NY : Longmans, Green and Co (1916).

17. Garkusha, I. et al. Journal of Nuclear Materials, 363-365 (2007), 1021-1025.

18. Bazylev, B. et al. Journal of Nuclear Materials, 390-391 (2009), 810-813.

19 Asano, K. et al. Contributions to Plasma Physics, 40 (2000), 3-4, 478-483.

20. Majeski, R. et al. Nuclear Fusion, 45 (2005), 6, 519.

21] K.Krieger. Journal of Nuclear Materials, (2011). PSI2010, private comm.

$22]$ Nedospasov, S. G., A.V.a. High Temperature, 48 (2010), 2, 285-287. Cited By (since 1996)

23. Pospieszczyk, A. et al. Journal of Physics B, 43 (2010), 14, 144017.

24. Bazylev, B. et al. Fusion Engineering and Design, 75-79 (2005), 407-411.

25. Bazylev, B. et al. Journal of Nuclear Materials, 363-365 (2007), 1011-1015.

26. Puetterich, T. et al. Nuclear Fusion, 50 (2010), 2, 025012.

27. Post, D. et al. Physics of Plasmas, 2 (1995), 6, 2328-2336.

28 Post, D. E. et al. Atomic Data and Nuclear Data Tables, 20 (1977), 5, 397 - 439.

29] Miloshevsky, G. and Hassanein, A. accepted by Nuclear Fusion, (2010).

[30] Bazylev, B. et al. Physica Scripta, 2009 (2009), T138, 014061.

[31] Shi, Y.; Miloshevsky, G. and Hassanein, A. Fusion Engineering And Design, (2010). 